\title{
OBITUARIO
}

\section{ANTONIO KRAPOVICKAS}

(1921-2015)

Nació en Buenos Aires el 8 de octubre de 1921, falleció en Corrientes el 17 de agosto de 2015.

Las personas que conocemos o tratamos a lo largo de nuestras vidas, entran muchas veces a formar parte de ella, se incorporan, influyen y dejan huella. El Ing. Antonio Krapovickas fue una de ellas, marcó vidas en forma indeleble.

Después de haber compartido con Krapovickas, durante más de medio siglo, los inicios y el crecimiento de su gran proyecto, el IBONE, crece la nostalgia por la ausencia de su generosa sabiduría, su cordialidad, su calidez, su respeto por las ideas, su humor inagotable. Fue un maestro, dispuesto siempre a ofrecer un consejo, a marcar valores éticos, a transmitir su enorme bagaje de conocimientos, su entusiasmo, dedicación y pasión por el trabajo. La manera

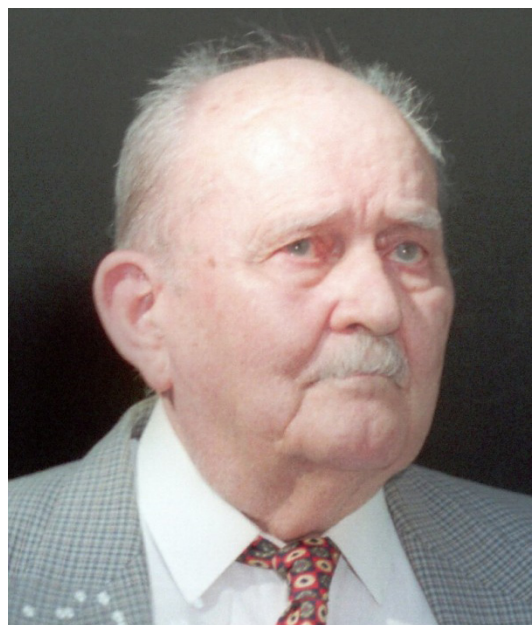
desinteresada en que brindó siempre su apoyo a colegas, estudiantes y a todas las personas que se acercaron a él o al Instituto de Botánica del Nordeste, lo hizo acreedor de su afecto.

Contar con su amistad fue un honor y un privilegio. Compartimos distendidas charlas, invariablemente amenas. En ellas afloraba su vena de erudito: transitaba desde una anécdota de la segunda guerra mundial, a la curiosa historia de algún tenor o al porqué de las costumbres de alguna comunidad aborigen. Fascinaba la amplitud de sus conocimientos y su memoria sorprendente.

Recientemente, cuando alguien le preguntó cómo querría que se lo recordara, contestó: "como un buen tipo". Y lo fue, honrado, amable, franco, alegre, magníficamente desprendido. Así lo recordaremos y siempre estará con nosotros.

La calidad de su producción científica, las numerosas distinciones humildemente recibidas, la creación, aunando esfuerzo y dedicación con su esposa, la Dra. Carmen Lelia Cristóbal, de una institución botánica de prestigio en el país y en el exterior y la formación de numerosos discípulos, lo colocan entre los grandes botánicos de su época. La joven generación tiene en el Ing. Antonio Krapovickas un espejo donde mirarse. A ella le corresponde tomar su bandera para perpetuar su legado.

Sara G. Tressens Instituto de Botánica del Nordeste

Tuve el honor de conocer al Ing. Agr. Antonio Krapovickas en agosto de 1973, al realizar un viaje a la Universidad Nacional del Nordeste, Corrientes, R.A., para recibir un entrenamiento en el estudio de la Familia Malváceas, campo de su especialidad. Al descender del avión me recibe una persona sonriente y amable y quedé sorprendida al descubrir que era todo lo contrario a lo imaginado. En efecto, una autoridad científica de prestigio internacional, como el ilustre científico Dr. Krapovickas, me acoge con toda sencillez sin hacer diferencia de nivel académico.

Mi estadía de seis meses en su Institución, me permitió valorar y admirar su dedicación en toda labor académica y de investigación que realizaba, siendo cuidadoso y muy crítico en sus publicaciones, trasmitiendo dichas cualidades a sus discípulos, con gran desprendimiento de compartir sus conocimientos con toda persona que requería una consulta; lo describen como una persona invaluable. 
Sus numerosas publicaciones de valioso contenido científico, lo destacan como la primera autoridad argentina en la ciencia botánica. La admiración y reconocimiento de botánicos nacionales y extranjeros a tan ilustre científico ha sido motivo para dedicarle nombres de géneros y especies nuevos para la ciencia.

Sus discípulos argentinos y del exterior que tuvimos la suerte de ser guiados por tan admirable maestro, científico y amigo, no dejaremos de extrañar sus pláticas y aportes al desarrollo de la Botánica, por ello me resta decir:

Ing. ANTONIO KRAPOVICKAS, CON EL CARIÑO, GRATITUD Y ADMIRACIÓN DE SIEMPRE, SU DISCÍPULA PERUANA LE DICE: MUCHAS GRACIAS Y HASTA SIEMPRE. Magda Chanco Estela Herbario San Marcos Universidad Nacional Mayor de San Marcos Lima, Perú

Trataré de recordar a Krapo, como todos lo llamábamos, evocando los agradables momentos en los que nos encontrábamos y podíamos hablar algún rato. También disfruté uno o dos días en su departamento de Corrientes, frente al río; una estancia muy agradable, con maníes y algún trago alcohólico, después de unas diez horas en el Instituto.

Recuerdo su famosa conferencia sobre las últimas y más extraordinarias novedades en Botánica, en unas Jornadas en La Plata, tiempo ha, donde todos estábamos expectantes por conocerlas. Finalmente fue una broma con gran ironía, sobre errores o ridiculeces publicadas; la presencié dos veces, la segunda con el agregado de un par de perlitas acerca de cactus con las que contribuí.

La dedicación completa del Ing. A. Krapovickas y de su esposa Carmen Cristóbal, se muestra en experiencias como la que narro a continuación. Sucedió en el viaje posterior a las Jornadas Argentinas de Botánica en 1985. Al llegar a la ciudad de Formosa, después de un día muy intenso de paradas para observar la vegetación, parecía no haber suficiente lugar para todos los participantes. El matrimonio Krapovickas nos guió hotel por hotel, pensiones, etc. hasta ubicar a los últimos alumnos. Luego de eso regresaron para que no faltáramos a la cena, donde el gobernador nos atendería, a la que en principio nadie pensaba ir, pero el ejemplo de ambos nos comprometió y allí estuvimos.

En lo profesional Krapovickas contribuyó con las Malváceas a la Flora de San Juan y, a diferencia de algunos principiantes, aceptó con modestia y agrado las preguntas y sugerencias de un novato como era yo respecto a él.

Estoy orgulloso de haber obtenido ese capítulo, donde Krapo puso todo su conocimiento, en especial del género Nototriche, que le interesaba mucho y del cual no existen claves generales; por lo menos está la de las especies de esa provincia.

En fin, dulces recuerdos y el dolor de que se haya ido.

\section{Roberto Kiesling \\ Centro Regional de Investigaciones $C$ y $T$ Instituto Argentino de Investigaciones de las Zonas Áridas Mendoza, Argentina.}

En memoria del Ing. Antonio Krapovickas

El Doctor Krapovickas no se ha ido. Contra lo inevitable dejó sus obras que fueron grandes y profundas, tanto que se continuarán en el tiempo. Además meritorias, que cuando ejecutadas eran tiempo de crisis, tanto en recursos como en comprensión. Esto por lo material y visible a lo que se debe agregar su imagen, eso que es impalpable, pero que quedó en el recuerdo de los que lo conocimos, que no son pocos y en quienes marcó huellas que continuarán presentes.

Pertenecía a un mundo cuyos componentes pertenecen al silencio, un silencio del que él también formaba parte, pero le agregó una discreta sobriedad. Así construyó sus obras manteniendo, como corresponde a un investigador, con crítica severa que defendía y aplicaba con un humor que tan bien lo caracterizaba; era su forma de ser espontánea y natural. 
Fue un místico de la investigación de un universo profundo y dinámico que necesitaba inteligencia para llegar hasta sus orígenes.

Vino a nuestro país varias veces, para los estudios florísticos y la genética, pero además transmitió un entusiasmo racional a los botánicos locales, que siguen agradeciéndolo. La ayuda que ofreció, permitió dar un paso grande hacia adelante. Aún ahora se continúa accediendo a su nido correntino, el IBONE, donde se encuentra herborizada una numerosa colección de la flora de este país, además de continuarse los intercambios de valiosa e indispensable información. Cada viaje es un pensamiento de recuerdo y agradecimiento que se mantienen aquí como una llama permanente.

\section{Marcos Sanjurjo K. Herbario "Teodoro Rojas" Facultad de Ciencias Químicas.- U.N.A. (FCQ) Asunción, Paraguay}

Ing. Antonio Krapovickas is recognized world-wide as THE authority regarding the taxonomy of the genus, Arachis. His contribution to the understanding of the genus will remain a firm basis for taxonomy, breeding and genetic research for many decades. Before my first meeting with Ing. Krapovickas, Dr. W.C. Gregory had informed me about the nature of the Botanist as well as the man. I quickly found that Dr. Gregory's words were true; Antonio was a true scholar and a gentleman. Over the years Antonio and I stayed in each other's homes when we visited, and we made five major plant exploration expeditions together. We shared data about the Arachis collections and we spent many days studying herbarium and discussing the traits of the accessions we had collected. Antonio was eager to learn of successes and failures I had in the cross-compatibility of the various Arachis materials. He was always willing to share the information he had accumulated on the wild Arachis and the cultivated accessions. Because we shared a common interest in the field and laboratory evaluation of the cultivated lines, we spent many days studying, evaluating and classifying those materials. Ing. Krapovickas will truly be missed in the years ahead.

Antonio was a dear friend and colleague and I am missing his presence daily.

\section{Texas Agricultural Experiment Station Charles Simpson
Experiment Station Texas A\&M University Stephenville, USA}

Estamos todos muy tristes por el fallecimiento de nuestro querido maestro Krapovickas. Uno sabe que el tiempo es inexorable y que nadie es físicamente eterno, pero es difícil aceptar la realidad. Hay una sensación profunda de que nos hace falta una pieza muy importante, la más importante, en la continuación de los trabajos con Arachis. Y ya nos hace falta poder disfrutar de su ejemplo de dedicación, de curiosidad científica y de la manera siempre alegre de vivir entre los amigos, con que él nos ha brindado. Su impacto en la vida de todos nosotros ha sido muy positivo y permanente.

Continuar trabajando juntos, entre nuestras instituciones y grupos, es seguramente lo mejor que podremos hacer para corresponder al legado.

\section{Jose Francisco Montenegro Valls Empresa Brasileira de Pesquisa Agropecuária Brasilia, DF, Brasil}

Tuve el honor de conocer a los Krapovickas en la década del 70, durante uno de mis primeros viajes de campo y visita al herbario de la Facultad de Agronomía en Corrientes. En ese momento, ya pude apreciar la enorme labor realizada, en corto tiempo, en lo que se convertiría en uno de los Institutos de Botánica más importantes de nuestro país. Además de su extraordinaria capacidad como científico, reconocida por sus excelentes trabajos en Malvacéas, en Arachis y en otros grupos, el Ing. Krapovickas, junto con Carmeta, llevaron a cabo una notable actividad de gestión 
y formación de recursos humanos, hoy plasmada en el IBONE y su gente. Posteriormente, pude compartir con ambos la creación y desarrollo de la Myndel Botanica Foundation en el año 2000; a partir de ese momento, trabajamos en la evaluación de becas y candidatos, por lo que pude apreciar el gran conocimiento, pasión y capacidad de análisis del Ingeniero por más de 15 años. Finalmente, durante las últimas Jornadas Argentinas de Botánica, realizadas en Salta, y durante la exposición de un video hecho en el IBONE sobre su vida, rescaté su deseo de ser recordado como "un buen tipo", lo que creo describe al Ing. Krapovickas en toda su dimensión.

Fernando Zuloaga

Instituto de Botánica del Darwinion San Isidro, Buenos Aires, Argentina

\section{Su trayectoria}

\section{Investigación}

El Ing. Agr. Antonio Krapovickas fue designado Investigador el 6 de diciembre de 1960 por el Directorio del CONICET, presidido por el premio nobel Bernardo Alberto Houssay.

Investigador Superior. Carrera del Investigador Científico y Tecnológico, CONICET, 1-I-1981 (ingreso: II-1961).

\section{Premios y distinciones:}

Beca John Simon Guggenheim Memorial Foundation, U.S.A., 1954.

Académico Correspondiente. Academia Nacional de Agronomía y Veterinaria, 15-X-1976.

Premio Konex 1983. "Diploma al Mérito", 9-IX-1983.

Premio CADIA 1984. Centro Argentino de Ingenieros Agrónomos, 3-VIII-1984.

Socio Honorario, Sociedad Argentina de Genética.

Socio Honorario, Sociedad Argentina de Botánica.

Corresponding Member. Botanical Society of America, 13-IX-1989.

Profesor Extraordinario. Universidad Nacional del Nordeste, 9-V-1990.

Premio Bunge y Born, en Agronomía. Fundación Bunge y Born, Buenos Aires, 17-VII-1990.

Presidente Honorario del VI Congreso Latinoamericano de Botánica. Mar del Plata, 2-8-X1994.

Premio CIA. Centro de Ingenieros Agrónomos de Gral. Cabrera y Zona. General Cabrera, prov. Córdoba, 28-IX-1995.

Categoría A, del Programa de Incentivos, Consejo Interuniversitario Nacional (CIN), X-1995. Académico. Academia Nacional de Ciencias, Córdoba, 16-VII-1997. Incorporación: 8-X-1998. Premio “Arturo Burkart”, Academia Nacional de Ciencias Exactas, Físicas y Naturales, 1997. Miembro de la Sociedad Científica del Paraguay, sesión 14-X-1997.

Miembro Honorario, Asociación de Biología de Tucumán, 5-X-2001.

Doctor Honoris Causae otorgado por la Universidad Nacional de Rosario, 16-VIII-2012.

\section{Gestión}

Fundador y Director del Instituto de Botánica del Nordeste - IBONE, convenio CONICETUNNE, Corrientes, (1977-2001).

Presidente de la Sociedad Argentina de Botánica (1972-74).

Presidente de la Sociedad Argentina de Genética (1983-85).

Presidente del Jurado del Premio Bunge y Born, atribuido a las Ciencias del Ambiente, Buenos Aires, 12-IV-1999.

Integrante del Tribunal para seleccionar al candidato al premio "Hermann Burmeister 1999", de la Academia Nacional de Ciencias en Córdoba. Córdoba, 15-XII-1999.

Miembro del Comité de Evaluación de la Myndel Botanical Foundation, (desde el 2001). 


\section{Docencia}

- Facultad de Ciencias Exactas, Fisicas y Naturales, Universidad Nacional de Córdoba.

1949-54, Profesor de "Genética".

1950, Profesor de "Botánica Sistematica II".

1951-53, Profesor de "Botánica" y "Fitogeografía".

- Facultad de Agronomía y Zootecnia, Universidad Nacional de Tucumán.

1957-60, Profesor de "Botánica".

1961-64, Profesor de "Genética".

- Escuela Superior de Ciencias Naturales, Universidad Nacional de Tucumán (Instituto Miguel Lillo). 1955-57, Profesor de "Anatomía Vegetal".

1957-62, Profesor de "Investigación del Departamento de Botánica".

1958-62, Profesor de "Citología y Genética".

- Facultad de Ciencias Agrarias, Universidad Nacional del Nordeste, Corrientes.

1964-67, Profesor Interino de "Genética y Fitotecnia".

1968-89, Profesor Titular de "Genética y Fitotecnia".

1989, se acoge a los beneficios de la jubilación.

9/5/1990, Profesor Extraordinario de la Universidad Nacional del Nordeste.

- Facultad de Ciencias Exactas, Físicas y Naturales, Universidad Nacional del Nordeste,

Corrientes.

1964-1989. Profesor "ad honorem" de "Genética y Biometría".

Sociedades a las que perteneció:

Sociedad Argentina de Botánica. Socio Honorario.

Sociedad Argentina de Genética. Socio Honorario

California Botanical Society. Socio Honorario.

Botanical Society of America. Corresponding Member.

Association for Tropical Biology.

Society for the Study of Evolution.

American Association for the Advancement of Science.

National Geographic Society.

Sociedad Científica del Paraguay.

\section{Trabajos publicados}

1.- Krapovickas, A. 1945. Notas sobre el género Modiolastrum en la Argentina. Revista Argent. Agron. 12(1): 38-44.

2.- Krapovickas, A. 1045. El género Neobaclea (Malvaceae) y su distribución geográfica en la República Argentina. Darwiniana 7(1): 108-112.

3.- Krapovickas, A. 1949. Las especies de Sphaeralcea de Argentina y Uruguay. Lilloa 17: 179-222.

4.- Krapovickas, A. 1949. Relación entre número cromosómico y área en el género Modiolastrum (Malvaceae). Lilloa 19: 121-125.

5.- Krapovickas, A. y V.A. Rigoni. 1949. Cromosomas de una especie silvestre de Arachis (Resumen). IDIA 24: 23-24. (Reimpreso en Arch. Fitotec. Uruguay 5(3): 406. 1953).

6.- Rigoni, V.A. y A. Krapovickas. 1949. Observaciones sobre herencia de color de testa de maní (Resumen). IDIA 24: 24. (Reimpreso en Arch. Fitotec. Uruguay 5(3): 406. 1953).

7.- Krapovickas, A. y V.A. Rigoni. 1950. Observaciones citológicas y genéticas en Arachis. Memoria $1^{\circ}$ Reunión de maní y girasol (Manfredi, 31-VIII-1950): 5-6. 
BONPLANDIA 24(2). 2015

8.- Krapovickas, A. 1950. Dos especies nuevas o críticas de Sphaeralcea (Malvaceae) de Perú. Bol. Soc. Argent. Bot. 3(2): 71-73.

9.- Krapovickas, A. 1950. Una nueva especie del género Nototriche (Malvaceae). Bol. Soc. Argent. Bot. 3(3): 170-173.

10.- Krapovickas, A. 1950. Revisión del género Lecanophora (Malvaceae). Darwiniana 9(2): 248-279.

11.- Krapovickas, A. 1951. Monteiroa, nuevo género de Malváceas. Bol. Soc. Argent. Bot. 3(4): 235-244.

12.- Krapovickas, A. 1951. Notas citotaxonómicas en Nototriche (Malvaceae) I. Bol. Soc. Argent. Bot. 4(1-2): 107-116.

13.- Krapovickas, A. 1951. Números cromosómicos de tres compuestas riojanas. Bol. Soc. Argent. Bot. 4(1-2): 105-106.

14.- Krapovickas, A. y V.A. Rigoni. 1951. Estudios citológicos en el género Arachis. Revista Invest. Agríc. 5(3): 289-294.

15.- Krapovickas, A. y A.M. Fuchs. 1952. Notas citológicas sobre Leguminosas. Darwiniana 9(3-4): 612-613.

16.- Krapovickas, A. 1952. Notas sobre Malváceas. Bol. Soc. Argent. Bot. 4(3): 187-191.

17.- Krapovickas, A. 1953. Notas citotaxonómicas sobre Nototriche (Malvaceae) II. Bol. Soc. Argent. Bot. 5(1): 51-74.

18.- Krapovickas, A. 1953. Malvaceae, en A.L.Cabrera, Manual de la flora de los alrededores de Buenos Aires: 303-312. Editoral Acme S.A. Buenos Aires.

19.- Krapovickas, A. y V.A. Rigoni. 1954. Cruzamientos interespecíficos en Arachis y consideraciones sobre el centro de origen del maní cultivado. Anais do $2^{\circ}$ Congresso Panamericano de Agronomía. Piracicaba, S.Paulo, Brasil. 266-267.

20.- Krapovickas, A. 1954. Sinopsis del género Tarasa (Malvaceae). Bol. Soc. Argent. Bot. 5(3): 113-143.

21.- Krapovickas, A. 1954. Estudio de las especies de Anurum, nueva sección del género Urocarpidium Ulbr. (Malvaceae). Darwiniana 10(4): 606-636.

22.- Krapovickas, A. y V.A. Rigoni. 1956. Noroeste argentino y Bolivia, probable centro de origen del maní cultivado. IDIA 106-108: 197.

23.- Krapovickas, A. y A.M. Fuchs. 1957. Notas citológicas sobre Leguminosas II. Revista Invest. Agric. 11(3): 215-218.

24.- Krapovickas, A. y V.A. Rigoni. 1957. Nuevas especies de Arachis vinculadas al problema del origen del maní. Darwiniana 11(3): 431-455.

25.- Krapovickas, A. 1957. Tres especies nuevas de Nototriche (Malvaceae) de Perú. Bol. Soc. Argent. Bot. 6(34): 233-238.

26.- Krapovickas, A. 1957. Números cromosómicos de Malváceas americanas de la tribu Malveae. Revista Agron. Noroeste Argent. 2(2): 245-260.

27.- Krapovickas, A. 1957. Notas sobre Malváceas II. Bol. Soc. Argent. Bot. 7(1): 37-41.

28.- Krapovickas, A. 1957. Las especies de Malvastrum sect. Malvastrum de la Flora Argentina. Lilloa 28: 181-195.

29.- Krapovickas, A. 1957. Sobre las especies polígamas de Nototriche (Malvaceae). Lilloa 28: 269-277.

30.- Krapovickas, A. 1960. Poliploidía y área en el género Tarasa (Malvaceae). Lilloa 30: 233-249.

31.- Krapovickas, A. 1960. Calyculogygas, nuevo género de Malváceas de Uruguay. Lilloa 30: 251-256.

32.- Krapovickas, A. 1960. Consideraciones sobre el origen del maní cultivado. IDIA 145: 69-71.

33.- Krapovickas, A. y W.C. Gregory. 1960. Arachis Rigonii, nueva especie silvestre de maní. Revista Invest. Agric. 14(2): 157-160.

34.- Krapovickas, A. 1960. Hibridación introgresiva en maníes de la región guaranítica argentina. Revista Invest. Agric. 14(2): 161-175.

35.- Rigoni, V.A., A. Krapovickas y J.R. Pietrarelli. 1960. Las variedades cultivadas de maní en la provincia de Córdoba. Revista Invest. Agric. 14(2): 177-196.

36.- Krapovickas, A. y V.A. Rigoni. 1960. La nomenclatura de las subespecies y variedades de Arachis hypogaea. Revista Invest. Agric. 14(2): 197-228.

37.- Dos especies nuevas de Monteiroa (Malvaceae) del estado de Santa Catarina. Sellowia 14: 61-65. 1962.

38.- Krapovickas, A. y C.L. Cristóbal. 1962. Notas sobre la sección Lebretonia, Pavonia (Malvaceae) y revisión de las especies argentinas. Lilloa 31: 5-74.

39.- Krapovickas, A. 1965. Notas sobre Malváceas III. Kurtziana 2: 113-126. 
40.- Krapovickas, A. y C.L. Cristóbal. 1965. Revisión del género Peltaea (Malvaceae). Kurtziana 2: 135-216.

41.- Krapovickas, A. 1965. Recuentos cromosómicos de Leguminosas. Kurtziana 2: 91-94.

42.- Krapovickas, A. 1965. Malvaceae, en A.L. Cabrera, Flora prov. Buenos Aires 4: 169-220.

43.- Krapovickas, A. 1967. Notas citotaxonómicas sobre Malveae. Kurtziana 4: 29-37.

44.- Krapovickas, A. 1969. Notas citotaxonómicas sobre Malváceas. Bonplandia 3(2): 9-24.

45.- Krapovickas, A. 1968. Origen, variabilidad y difusión del maní (Arachis hypogaea). Actas y Memorias 37 Congreso Internacional de Americanistas 2: 517-534. La versión inglesa se publicó en "The domestication and exploitation of plants and animals" ed. P.J. Ucko y C.W. Dimbleby. London, 1969, pgs. 427-441.

46.- Krapovickas, A. 1969. Notas sobre el género Abutilon Mill. (Malvaceae) I, La sección Tetrasida (Ulbr.) Krap. Bonplandia 3(3): 25-47.

47.- Krapovickas, A. 1969. Una nueva especie patagónica de Lecanophora Speg. (Malvaceae). Revista Fac. Ci. Agrar. Univ. Nac. Cuyo 14(1): 36-38.

48.- Seeligmann, P y A. Krapovickas. 1969. Current chemotsystematic studies in Arachis. Chem. Pl. Taxonomy Newsletter 13: 8.

49.- Krapovickas, A. 1970. El género Malvella Jaub. et Spach (Malvaceae) en Argentina. Bonplandia 3(5): 53-62.

50.- Krapovickas, A. 1970. Malváceas nuevas sudamericanas. Bonplandia 3(6): 63-72.

51.- Krapovickas, A. 1970. Historia de la Botánica en Corrientes. Bol. Soc. Argent. Bot. 9, Suplemento: 229-275.

52.- Krapovickas, A. 1970. Dos géneros nuevos de Malváceas: Diramphis y Hochreutinera, con notas sobre los afines Briquetia y Neobrittonia. Darwiniana 16(1-2): 219-232.

53.- Krapovickas, A. 1971. Influencia de la humedad y de la alternancia de temperatura en la germinación en especies del género Arachis (Leguminosae). Oleagineux 26(7): 469-474.

54.- Krapovickas, A. 1971. Evolución del género Tarasa (Malvaceae). Recientes adelantos en Biología, págs. 232-241, Bs. Aires.

55.- Krapovickas, A. 1972. La información cromosómica y su importancia en la sistemática. Memorias de Symposio del I Congreso Latinoamericano de Botanica, págs. 247-264, México.

56.- Gregory, W.C., M. Pfluge Gregory, A. Krapovickas, B.W. Smith y J.A. Yarbrough. 1973. Structure and genetic Resources of Peanuts. "Peanuts - Culture and Uses, A Symposium", Stillwater, Oklahoma (1 vol. 684 págs.), págs. 47-133.

57.- Krapovickas, A. 1973. Las especies de Nototriche publicadas por Cavanilles en "Icones et Descriptiones Plantarum”. Bol. Soc. Argent. Bot. 15(2): 285-286.

58.- Krapovickas, A. 1973. Evolution of the genus Arachis. Agricultural Genetics, Selected Topics, ed. B. Noav, J.Wiley y Sons, New York-Jerusalem, págs. 135-151.

59.- Krapovickas, A. 1974. Acaulimalva, nuevo género de Malváceas. Darwiniana 19: 9-39. 1974.

60.- Krapovickas, A. 1974. Malvastrum grandiflorum, nueva especie de Malvacea de Bolivia. Bol. Soc. Argent. Bot. 15(4): 467-470.

61.- Krapovickas, A. 1974. Luehea crispa, nueva especie de Tiliacea de Brasil. Bol. Soc. Argent. Bot. 16(1-2): 89-92.

62.- Krapovickas, A., S.G. Tressens y A. Fernández. 1974. Gaya elingulata, nueva especie de Malvacea de Brasil. Bol. Soc. Argent. Bot. 16(1-2): 93-96.

63.- Krapovickas, A., A. Fernández y P. Seeligmann. 1974. Recuperación de la fertilidad en un híbrido interespecífico estéril de Arachis. Bonplandia 3(11): 129-142.

64.- Krapovickas, A. 1975. Estudio y conservación del germoplasma en el género Arachis. Reunión Nacional sobre problemas genéticos en el mejoramiento vegetal y animal, Cerrillos, mayo 1975. Facultad de Agronomía y Zootecnia, Tucumán, Miscelánea 54: 9-12.

65.- Krapovickas, A. 1977. Sinopsis de la sección Lebretonia del género Pavonia (Malvaceae). XXVI Congresso Nacional de Botânica, Rio de Janeiro, 1975, págs. 307-322.

66.- Krapovickas, A. 1978. Una nueva especie de Pavonia (Malvaceae) del estado de Minas Gerais (Brasil). Revista Brasil. Bot. 1: 89-91.

67.- Krapovickas, A. 1978. Malvaceae, en Steyermark, J.A. y O. Huber, Flora de Avila (Venezuela): 589-598. Caracas.

68.- Krapovickas, A. y J.R. Pietrarelli. 1978. Maní, vulnerabilidad y recursos genéticos. Ciencia e Investigación 34 (7-10): 128-131.

69.- Krapovickas, A. 1979. Novedades sobre Tarasa (Malvaceae). Bol. Soc. Bot. La Libertad 11(1-2): 43-49. 
BONPLANDIA 24(2). 2015

70.- Gregory, W.C., A. Krapovickas y M. Pfluge Gegory. 1980. Structure, Variation, Evolution and Classification in Arachis. Advances in Legume Sciences, edited by W.J.Summerfield y A.H.Bunting: 469-481. Kew.

71.- Krapovickas, A. 1982. Dos especies nuevas de Abutilon Mill. (Malvaceae) de Brasil. Hickenia 1(51): 269276.

72.- Krapovickas, A. 1982. Novedades en Pavonia Cav. sect. Typhalaea Gueke (Malvaceae). Bol. Soc. Argent. Bot. 20(3-4): 281-301.

73.- Krapovickas, A. 1982. Novedades en Pavonia Cav. sect. Goetheoides Guerke (Malvaceae. Anais XXXII Congresso Nacional de Botânica, Teresina, Piaui, Brasil, págs. 67-84.

74.- Krapovickas, A. 1983. Notas sobre Malváceas, IV. Bonplandia 5(27): 257-273.

75.- Krapovickas, A. 1984. Malvaceae, en Claves de los géneros de Fanerógamas, ed. A.T. Hunziker. Bol. Soc. Argent. Bot. 23(1-4): 180-185.

76.- Fryxell, P.A.. A. Krapovickas y D. Crewz, 1984. Sidus sidarum - IV. A new record of Sida in North America, S. santaremensis (Malvaceae). Sida 10(4): 319-320.

77.- Valls, J.F.M., V. Ramanatha Rao, C.E. Simpson y A. Krapovickas. 1985. Current status of collection and conservation of South American groundnut germplasm with emphasis on wild species of Arachis. Proceedings of an international workshop on cytogenetics of Arachis, 31 oct. - 2 nov. 1983. India, ICRISAT, págs. 15-35.

78.- Krapovickas, A. 1985. Nuevas combinaciones en Malváceas. Bol. Soc. Argent. Bot. 24(1-2): 205-207.

79.- Krapovickas, A. y C.L. Cristóbal. 1985. El género Peltaea (Malvaceae) en Argentina. Bol. Soc. Argent. Bot. 24(1-2): 207.

80.- Fryxell, P.A. y A. Krapovickas. 1986. Proposal to conserve 5007A Peltaea against Peltostegia (Malvaceae). Taxon 35(2): 389- 390.

81.- Krapovickas, A. 1988. Malvaceae, en M.N. Correa, Flora Patagónica 5: 126-153.

82.- Krapovickas, A., P. A. Fryxell y D. M. Bates. 1988. Allosidastrum, un nuevo género de Malvaceae de los Neotropicos. Bol. Soc. Bot. Méx. 48: 23-34.

83.- Fryxell, P.A. y A. Krapovickas. 1990. The Malvaceae published by Turczaninow. Contr. Univ. Mich.Herb. 17: 173-182.

84.- Krapovickas, A. y S. Cáceres Moral. 1990. La identidad del género Melinia (Asclepiadaceae). Darwiniana 30(1-4): 277-280.1990.

85.- Krapovickas, A. 1990. Dos especies nuevas de Pavonia Cav. (Malvaceae) de Brasil. Anais do XXXVI Congresso Brasileiro de Botanica. Sociedade Botânica do Brasil - Curitiba - 1985. Vol.1: 281-286.

86.- Krapovickas, A. y C.L. Cristóbal. 1993. Novedades en Malváceas Sudamericanas. Bonplandia 7(1-4): 4556.

87.- Krapovickas, A. y P.A. Fryxell. 1993. Algunos tipos de Malváceas descriptos por C.B.Presl. Bonplandia 7(1-4): 57-62.

88.- Pool, A. y L.Brako; reviewed by A.Krapovickas. 1993. Malvaceae, Prepared by A.Pool (MO) y L. Brako; reviewed by A.Krapovickas (CTES), with additional help of M.Chanco (USM). En Catálogo de las Angiospermas y Gimnospermas del Perú, por L. Brako y J.L. Zarucchi.

89.- Leites Bueno, O. y A. Krapovickas. 1994. Malvaceae - novas ocorrencias para o Rio Grande do Sul e Brasil. Heringia, Ser. Bot. Porto Alegre 44: 3-14.

90.- Krapovickas, A. y W.C. Gregory. 1994. Taxonomía del género Arachis (Leguminosae). Bonplandia 8(1-4): 1-186.

91.- Fernández, A. y A. Krapovickas. 1994. Cromosomas y evolución en Arachis (Leguminosae). Bonplandia 8(1-4): 187-220.

92.- Grosso, N.R., A. Krapovickas, J.R. Pietrarelli y C.A. Guzmán. 1994. Las proteínas seminales del maní (Arachis hypogaea, Leguminosae) Bonplandia 8(1-4): 221-233.

93.- Lopes Esteves, G. y A. Krapovickas. 1995. Duas especies de Pavonia Cav. (Malvaceae) do sul da Bahia, Brasil. Hoehnea 22(2): 41-45.

94 - Krapovickas, A. 1995. El origen y dispersión de la variedades del Maní. Academia Nacional de Agronomía y Veterinaria 49(12): 18-26.

95.- Krapovickas, A. 1996. Sinopsis del género Gaya (Malvaceae). Bonplandia 9(1-2): 57-87.

96.- Krapovickas, A. 1996. La identidad de Wissadula amplissima (Malvaceae). Bonplandia 9(1-2): 89-94.

97.- Krapovickas, A. y C.L. Cristóbal. 1996. Peltaea obsita (Mart. ex Colla) Krapov. y Cristóbal nov. comb. Bonplandia 9(1-2): 148. 
98.- Krapovickas, A. 1996. El origen y dispersión de las variedades del maní. Acad. Nac. Agron. Vet., 49: 18-26.

99.- Krapovickas, A. 1996. Problemas en la identificación de especies vegetales: el caso Arachis. Mendeliana 12(1): 10-13.

100.- Krapovickas, A. 1996. Agricultura indígena en las llanuras de la Cuenca del Plata. Acad. Nac. Agron. Vet., 50(17): 31-45.

101.- Krapovickas, A. y C.L. Cristóbal. 1997. La tipificación de Malva spicata L. (Malvaceae). Bonplandia 9(34): 257-258.

102.- Krapovickas, A. 1997. Los primitivos recolectores de germoplasma en el Nuevo Mundo. Ciencia e Investigación 50 (1-2): 45-47.

103.- Krapovickas, A. 1998. La colaboración internacional en recursos genéticos: el caso del maní (Arachis L.: Leguminosae). Proceedings of the VI Congreso Latinoamericano de Botánica. Missouri Bot. Garden Press.

104.- Krapovickas, A. 1999. Arachis hypogaea var. hirsute y las relaciones transoceánicas precolombinas. Anal. Acad. Nac. Cs. Ex. Fís. y Nat. 50: 211-216.

105.- Krapovickas, A. 1999. Convolvulaceae. Catálogo de las Plantas Vasculares de la Argentina. Monogr. Syst. Bot. Missouri Bot. Gard. 74: 547-565.

106.- Krapovickas, A. 1999. Fabaceae (Arachis). Catálogo de las Plantas Vasculares de la Argentina. Monogr. Syst. Bot. Missouri Bot. Gard. 74: 643-645.

107.- Krapovickas, A. 1999. Malvaceae. Catálogo de las Plantas Vasculares de la Argentina. Monographs Systematic Botany, Missouri Bot. Gard. 74: 813-844.

108.- Krapovickas, A. 1998. Arachis hypogaea var. hirsuta y las relaciones transoceánicas precolombinas. Anal. Acad. Nac. Cs. Ex. Fís. y Nat., Buenos Aires, 50: 211-216.

109.- Veiga, R.F. de A., J.F.M. Valls, C.R. Lopes, P.R. Curi y A. Krapovickas. 1999. Caracterição morfologica e agronômica de populaciones de Arachis sylvestris (A. Chev.) A. Chev. Boletim Cientifico (Instituto Agronômico, Campinas, SP, Brasil) 47: 1-28.

110.- Simpson, C.E., A. Krapovickas y J.F.M. Valls. 2001. History of Arachis including evidence of A. hypogaea L. progenitors. Peanut Science 28: 78-80.

111.- Esteves, G.L. y A. Krapovickas. 2002. New species of Abutilon (Malvaceae) from São Paulo State (Brazil). Kew Bull. 57: 479-482.

112.- Krapovickas, A. 2003. Las especies austroamericanas del género Cienfuegosia Cav. (MalvaceaeGossypieae). Bonplandia 12 (1-4): 5-47.

113.- Krapovickas, A. 2003. Revisión del género Monteiroa Krapov. (Malvaceae-Malveae). Bonplandia 12 (14): 49-62.

114.- Krapovickas, A. 2003. Tropidococcus Krapov., nuevo género de Malváceas. Bonplandia 12 (1-4): 63-66.

115.- Krapovickas, A. 2003. Sida sección Distichifolia (Monteiro) Krapov. comb. nov., stat. nov. (MalvaceaeMalveae). Bonplandia 12 (1-4): 83-121..

116.- Krapovickas, A. 2003. Revisión de Sida sección Muticae C.Presl (Malvaceae-Malveae). A. Krapovickas. Bonplandia 12 (1-4): 123-132.

117.- Krapovickas, A. 2003. Bordasia Krapov., nuevo género de Malváceas. Bonplandia 12 (1-4): 133-135.

118.- Fernández, A., A. Krapovickas, G. Lavia y G. Seijo. 2003. Cromosomas de Malváceas. Bonplandia 12 (1-4): 141-145.

119.- Krapovickas, A. 2003. Malvaceae, Malváceas. En: R. Kiesling. Flora de San Juan 2: 105-135.

120.- Fryxell, P.A. y A. Krapovickas. 2004. Six new species of Bolivian Hibiscus (Malvaceae). Novon 14(1): 58-69.

121.- Krapovickas, A. 2004. Las especies de Pseudabutilon con pelos glandulares en el cáliz (MalvaceaeMalveae). Bonplandia 13 (1-4): 31-34.

122.- Krapovickas, A. y P.A. Fryxell. 2004. Las especies sudamericanas de Hibiscus secc. Furcaria (MalvaceaeHibisceae). Bonplandia 13 (1-4): 35-115.

123.- Seijo, G., G.L. Lavia, A. Fernández, A. Krapovickas, D. Ducasse y E.A. Moscone. 2004. Physical mapping of the 5S and 18S-25S rRNA genes by Fish as evidence that Arachis duranensis and A. ipaensis are the wild diploid progenitors of A. hypogaea (Leguminosae). Amer. J. Bot. 9(9) 1294-1303.

124.- Krapovickas, A. 2004. Consideraciones prehistóricas sobre el origen del maní cultivado. Academia Nacional de Agronomía y Veterinaria 58: 320-331.

125.- Valls, J.F.M. y C.E. Simpson. 2005. New species of Arachis (Leguminosae) from Brazil, Paraguay and 
BONPLANDIA 24(2). 2015

Bolivia. Bonplandia 14 (1-2): 35-63.

126.- Krapovickas, A. 2005. Malvaceae, en A.Burkart y N.M. Bacigalupo, Flora Ilustrada de Entre Ríos, IV: 265-333.

127.- Krapovickas, A. 2006. Las especies argentinas y de países vecinos de Sida secc. Nelavaga (Malvaceae, Malveae). Bonplandia 15 (1-2): 5-45.

128.- Krapovickas, A. 2006. Dos especies nuevas de Hibiscus secc. Furcaria (Malvaceae) de Minas Gerais (Brasil) Bonplandia 15 (1-2): 47-51.

129.- Krapovickas, A. 2006. Novedades sudamericanas en Acaulimalva y Gaya (Malvaceae, Malveae). Bonplandia 15 (3-4): 103-112.

130.- Krapovickas, A. 2006. El tipo de Wissadula macrantha R.E. Fr. (Malvaceae, Malveae). Bonplandia 15 (3-4): 121-123.

131.- Krapovickas, A. 2007. Novedades en el género Sida (Malvaceae, tribu Malveae). Bonplandia 16 (3-4): 193-208.

132.- Krapovickas, A. 2007. Las especies de Sida secc. Malachroideae (Malvaceae) del Cono Sur de Sudamérica. Bonplandia 16 (3-4): 209-253.

133.- Krapovickas, A. 2007. El tipo de Trithrinax campestris (Palmae). Bonplandia 16 (3-4): 271-273.

134.- Lavia, G.I., A. Fernández y A. Krapovickas. 2007. Cromosomas de especies americanas de Sida (Malvaceae). Bonplandia 16 (3-4): 255-258.

135.- Krapovickas, A. y W.C. Gregory. 2007. Taxonomy of the genus Arachis (Leguminosae). Translated by D.E. Williams y C.E. Simpson. Bonplandia 16 (Supl.): 1-205.

136 - Seijo, J.G., G.I. Lavia, A. Fernández, A.Krapovickas, D.Ducasse, D.J. Bertioli y E.A. Moscone. 2007.

Genomic relationships between the cultivated peanut (Arachis hypogaea - Leguminosae) and its close relatives revealed by double GISH. Amer. J. Bot. 94: 1963-1971.

137.- Krapovickas, A. 2008. Nuevas especies de Malvaceae. Bonplandia 17(1): 35-46.

138.- Krapovickas, A. y J. G. Seijo. 2008. Gossypium Ekmanianum (Malvaceae), algodón silvestre de la República Dominicana. Bonplandia 17(1): 55-64.

139.- Krapovickas, A. y M. Dematteis. 2008. Nota: Butia eriospatha (Drude) Becc., palmera naturalizada en el norte de Misiones (Argentina). Bonplandia 17(1): 91-92.

140.- Krapovickas A. y J.A. Tolaba 2008. Malvaceae Juss. Flora del Valle de Lerma, Aportes Botánicos de Salta, Serie Flora 8(12): 1-107.

141.- Krapovickas, A. Malvaceae. 2009. En: F.O. Zuloaga, O.N. Morrone y M.J. Belgrano (eds.) Cat. Pl. Vasc.

Cono Sur, Monogr. Syst. Bot. Missouri Bot. Gard., St. Louis. 107(3): 2463-2520.

142.- Krapovickas, A. 2009. Spirabutilon Krapov., nuevo género de Malváceas (s.str.). Bonplandia 18(1): 25-28.

143.- Krapovickas, A. y R. O. Vanni. 2009. El maní de Llullaillaco. Bonplandia 18(1): 51-56.

144.- Krapovickas A. 2009. Novedades en Convolvuláceas argentinas. Bonplandia 18(1): 57-64.

145.- Lopes Esteves, G. y A. Krapovickas. 2009. Flora de Grão-Mogol, Minas Gerais: Malvaceae. Bol. Bot. Univ. São Paulo 27(1): 63-71.

146.- Krapovickas, A. 2010. Nuevas especies de Pavonia sección Malvaviscoides (Malvaceae) de Minas Gerais, Brasil. Bonplandia 19(1): 31-45.

147.- Krapovickas, A. 2010. Malvaceae varia. Bonplandia 19(1): 79-89.

148.- Krapovickas, A. 2010. Las ilustraciones de la «Historia General y Natural de las Indias, Islas y Tierrafirme del mar Océano de Gonzalo Fernández de Oviedo y Valdéz». Bonplandia 19(1): 91-96.

149.- Krapovickas, A. 2010. Novedades en Pavonia sect. Malvaviscoides (Malvaceae). Bonplandia 19(2): 127133.

150.- Krapovickas, A. 2010. Maníes arqueológicos de Argentina. Bonplandia 19(2): 183-192.

151.- Krapovickas A. 2010. La domesticación y el origen de la agricultura. Bonplandia 19(2): 193-199.

152.- Grings, M., A. Krapovickas y I.L. Boldrini. 2011. A new species of Pavonia (Malvaceae) from Southern Brazil. Syst. Bot. 36(2): 419-423.

153.- Krapovickas, A. 2011. Novedades en el género Malvastrum (Malvaceae). Bonplandia 20(1): 55-72.

154.- Krapovickas, A. 2011. Artículo invitado: Sembrar, plantar, cultivar, domesticar. Bonplandia 20(2): 419-426.

155 - Grings, M., A. Krapovickas y I.I. Boldrini. 2011. A new species of Pavonia (Malvaceae) from Southern

Brazil. Syst. Bot. 36(2): 419-423.

156.- Krapovickas, A. 2012. Novedades en Pavonia (Malvaceae). Bonplandia 21(1): 61-70. 
157.- Krapovickas, A. 2012. Novedades en el género Gaya (Malvaceae). Bonplandia 21(1): 71-76.

158.- Krapovickas, A. 2012. Novedades en las secciones Cordifoliae, Distichifolia, Malacroidea, Muticae y Nelavaga del género Sida (Malvaceae). Bonplandia 21(1): 77-92.

159.- Krapovickas, A., R. O. Vanni, J. R. Pietrarelli y C. E. Simpson. 2013. Las razas de maní de Perú. Bonplandia 22(1): 19-90.

160.- Krapovickas, A. 2013. Hibiscus Paulae (secc. Furcaria), nueva especie de Malvaceae de Minas Gerais (Brasil). Bonplandia 22(2): 137-139.

161 - Krapovickas, A. 2014. ¿Qué pasó ahí? El caso del maní. Revista Ciencia e Investigación, Reseñas 2(1): 27-32.

162 - Krapovickas, A. 2014. Nuevas especies de Sida, sección Sida (Malvaceae). Bonplandia 23(2): 65-118.

163 - Krapovickas, A. 2015. Notas sobre Urocarpidium y Fuertesimalva (Malvaceae). Bonplandia 24(1): 43-49.

164.- Krapovickas, A. 2015. Una nueva especie de Malvastrum (Malvaceae) de La Rioja (Argentina). Bonplandia 24 (2): 77-80. 
